\title{
¿Qué hay de nuevo en la metacognición? Revisión del concepto, sus componentes y términos afines
}

\author{
Ángel Valenzuela M. ${ }^{1}$ \\ https://orcid.org/0000-0002-4046-805X
}

\section{Resumen}

La investigación sobre metacognición cumple un poco más de cuatro décadas. Pese a ello, aún existen discrepancias acerca de su definición y los mecanismos de procesamiento involucrados en ella. Por esto, el objetivo del presente estudio es hacer una revisión actualizada del concepto, funcionamiento y componentes del proceso de metacognición. En cuanto a la metodología, se efectuó un recorrido histórico por las investigaciones sobre la temática, con el fin de configurar un marco conceptual actualizado. Se revisaron, principalmente, las bases de datos Scopus, WoS y SCielo posteriores al año 2000 utilizando como palabras clave metacognición y estrategias metacognitivas. Se intenta, de esta manera, responder a preguntas: ¿cómo se ha definido la metacognición durante estos últimos años? y ¿qué se dice sobre los componentes, subcomponentes y términos afines? En cuanto a la propuesta conceptual, se hace hincapié en señalar que esta es un proceso cognitivo de segundo orden, el cual se activa bajo ciertas condiciones (ejemplo: tareas complejas como lectura y escritura en educación superior) con el fin de monitorear y regular los procesos cognitivos. Sobre los componentes, se conservan los propuestos por Flavell y Brown: conocimiento y regulación de la cognición. Sin embargo, se suman otros conceptos que son claves en la investigación en metacognición, por ejemplo, juicios metacognitivos, estrategias metacognitivas, habilidades metacognitivas, autorregulación, entre otros. En conclusión, la metacognición es, sin lugar a duda, un campo de estudio de singular valor en el que aún existen interrogantes por esclarecer.

\section{Palabras clave}

Metacognición - Componentes de la metacognición - Teorías de la metacognición.

1- Universidad de Talca, Talca, Chile. Contacto: anvalenzuela@utalca.cl.

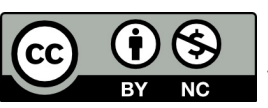

DOl: http://dx.doi.org/10.1590/S1678-4634201945187571

This content is licensed under a Creative Commons attribution-type BY-NC. 


\title{
What's new in metacognition? Review of the concept, its components and related terms
}

\begin{abstract}
Research on metacognition is around four decades old. However, there are still discrepancies about its meaning and the processing mechanisms involved. Therefore, the objective of this review is to clarify the concept, how it works, and its components. In order to do so, a historical tour of the research on this topic was carried out, which allowed to configure an updated conceptual framework that accounts for the theoretical proposals on the concept, its components and the terms related to it. Basically, Scopus, WoS and SCielo databases after 2000 were reviewed using metacognition and metacognitive strategies as key words. My attempt is to answer the questions: how has metacognition been defined over the last years? And what is said about the components, subcomponents, and related terms? Regarding the conceptual proposal, it is emphasized that this is a second-order cognitive process, which is activated under certain conditions (eg., complex tasks such as reading and writing in higher education) in order to monitor and regulate cognitive processes. On the components, those proposed by Flavell and Brown are preserved: knowledge and regulation of cognition. However, other key concepts are added (metacognitive judgments and their different varieties). In conclusion, metacognition is undoubtedly a relevant research field in which there are still questions to be clarified.
\end{abstract}

\section{Keywords}

Metacognition - Knowledge of cognition - Regulation of cognition.

\section{Presentación}

El término metacognición se introdujo por primera vez por John Flavell en el capitulo Metacognitive aspects of problem solving del libro The nature of intelligence, publicado en 1976. En ese capítulo se señala que este pensar sobre el pensar corresponde al conocimiento que un individuo tiene sobre sus propios procesos y productos cognitivos y sobre los aspectos que influyen en dicho proceso: ambiente, tarea y habilidades para resolver esa tarea. Desde entonces, la investigación ha posibilitado un sinfín de teorías, en ámbitos como la psicología cognitiva y la educación ${ }^{2}$.

\footnotetext{
2- Otros ámbitos específicos también han indagado en la metacognición: interacciones sociales (HEYES, 2016; SCHNEIDER, 2008); comprensión de los procesos ejecutivos (KORIAT, 2012); identificación de la naturaleza de la memoria (MAZZONI; SCOBORIA; HARVEY, 2010); comprensión lectora y producción de textos (ESCORCIA et al., 2017; ESCORCIA; FENOUILLET, 2011; PERONARD, 2002b; VEENMAN, 2016); teoría sobre los juegos de azar (LUEDDEKE; HIGHAM, 2011); diferencias cognitivas entre especies humanas y no humanas (VINING; MARSH, 2015); enfermedades mentales (GARAY; KEEGAN, 2016; HAMM; LYSAKER, 2016); reconocimiento facial (KELLY; METCALFE, 2011), entre otros.
} 
En estos más de 40 años de investigación sobre metacognición, los principales hallazgos dan cuenta de que sujetos que exhiben niveles más altos de conocimiento y regulación metacognitiva tienden a desarrollar un aprendizaje más exitoso (BROWN et al., 1982; FLAVELL, 1979; GUTIERREZ et al., 2016; VEENMAN; VAN HOUT-WOLTERS; AFFLERBACH, 2006). Además, se ha demostrado que el desarrollo metacognitivo actúa como variable predictora del rendimiento en situaciones de aprendizaje tanto como dominio específico y como dominio general (BINBASARAN TUYSUZOGLU; GREENE, 2015; NATIONAL READING PANEL, 2000; VEENMAN, 2008, 2016; WANG; HAERTEL; WALBERG, 1993).

Sin embargo, a pesar de la cuantiosa literatura especializada y de los hallazgos, aún surgen cuestionamientos sobre la definición conceptual y operacional, sobre las características que se le atribuyen, y sobre los componentes que en ella interactúan (SCHUNK, 2008; VEENMAN, 2016). Esto hace que se vuelva un concepto opaco y nebuloso, afectando indudablemente las mismas investigaciones. Ante esto, no es de extrañar que las conclusiones en estudios sobre metacognición, a menudo, sean inconsistentes cuando se han utilizado diferentes definiciones y medidas, las que en ocasiones parecen contradictorias.

Por ello, en la presente revisión se pretende configurar un marco conceptual actualizado, que dé cuenta de las propuestas teóricas del concepto, los componentes y los términos afines a la metacognición. Por esto, la investigación consistió en hacer una revisión de la literatura durante las últimas dos décadas con el fin de responder a las interrogantes: ¿cómo se ha definido la metacognición durante estos últimos años? y ¿qué se dice sobre los componentes, subcomponentes y términos afines?

\section{Aspectos iniciales y desarrollo histórico del concepto}

Flavell al proponer el concepto metacognición, enfatiza en las propiedades del prefijo meta (sobre, o más allá, o más arriba), aludiendo a la capacidad de pensar sobre el pensamiento (FLAVELL, 1976, 1979) o de una cognición sobre la cognición (FLAVELL, 1992; WELLMAN, 1985), la cual consiste en el monitoreo, regulación y orquestación de los procesos cognitivos al servicio de una meta u objetivo (FLAVELL, 1976). No obstante, con anterioridad a Flavell ya existía una preocupación por este tipo de procesos. Por ejemplo, en Aristóteles, Descartes, Dewey o en James, hay una clara alusión a la importancia de los procesos cognitivos internos como acción que guía el conocimiento y el aprendizaje (MARTÍ, 1995; NELSON; NARENS, 1994).

Pese a ello, no es hasta el surgimiento del cognitivismo, como reacción al conductismo, y principalmente a los aportes de Piaget y Vygotsky, que la investigación manifiesta una inquietud explícita por el mundo interior del hombre (FLAVELL, 1992; GALLEGOS et al., 2014; NELSON, 1996; PERONARD, 2002a, 2009; WELLMAN, 1985), desencadenando la necesidad de investigar los procesos subyacentes a la cognición. A partir de esto, Tulving y Madigan, a principios de los 70, refiriéndose al funcionamiento de los propios procesos de memoria, proponen el concepto de metamemoria (TULVING; MADIGAN, 1970; VEENMAN; VAN HOUT-WOLTERS; AFFLERBACH, 2006; WELLMAN, 1985), antecedente próximo de la metacognición. Así, cuando Flavell presenta un modelo 
de monitoreo y regulación de los procesos cognitivos, mantiene el uso del prefijo meta, surgiendo lo que conocemos como metacognición.

A partir de estas propuestas, destaca la distinción que se hace de la acción de conocer, explicitando que el hecho de poder acceder a los propios procesos cognitivos a través de esta operación permitiría mejorar el control sobre dicha actividad. Precisamente, los elementos clave que comprenden este primer modelo, son el conocimiento metacognitivo, las experiencias metacognitivas, el conocimiento de las metas o tareas y el conocimiento de las estrategias (FLAVELL, 1979). Posteriormente, Brown y otros autores (1982) sugieren la existencia de dos tipos de conocimiento metacognitivo: un conocimiento declarativo, referido a la persona, a la tarea, y a la estrategia; y un conocimiento procedimental, ligado a la regulación de los procesos cognitivos durante la planificación, control y evaluación, vale decir, conocer cómo se realiza lo que se realiza.

A mediados de los 80, Brown (1987) modifica el modelo propuesto inicialmente por Flavell, bajo el supuesto de que el conocimiento de la cognición no garantizaría su regulación ${ }^{3}$ y presenta un modelo alterno, en donde el conocimiento metacognitivo se configura de manera autónoma a la regulación de la cognición. En ese sentido, a pesar de estar conectados cada componente, cada uno alimentando al otro, son claramente distinguibles. Así, sobre el conocimiento de la cognición, Brown (1987) señala que corresponde a la información que la gente tiene acerca de sus propios procesos cognitivos, basándose en el supuesto de que cualquier sujeto puede retroceder y/o reconsiderar estos como objetos de pensamiento y reflexión. La regulación de la cognición, en cambio, consistiría en los procedimientos utilizados para regular y supervisar el aprendizaje a través de la planificación, el monitoreo durante el acto de aprender, y la evaluación de la eficiencia y eficacia de los resultados de las acciones realizadas (BROWN, 1987). En esta propuesta destaca el énfasis que le da a los procesos ejecutivos, subrayando la importancia del control que las personas aportan o no, a los esfuerzos cognitivos.

En la década de los 90 resaltan los hallazgos que refuerzan el carácter predictivo de la metacognición en el aprendizaje y en el desarrollo de la autonomía (WANG; HAERTEL; WALBERG, 1993). No obstante, se cuestiona la enseñanza explícita y deliberada de esta en contextos de aprendizaje, ya que según Nickerson, Perkins y Smith (1994), las personas no aprenden cómo aprender como resultado de un esfuerzo deliberado por enseñar esa habilidad de modo explícito y consciente, sino que el aprendizaje se da de manera contextual y relacionada a otros saberes y habilidades. De este modo, cuestionan la aplicación de programas educativos centrados en desarrollar la metacognición sin la base de un contexto. Por otro lado, resalta la especificación que propone Schraw y Dennison (1994) sobre el conocimiento de la cognición, estableciendo tres tipos: a) un conocimiento declarativo, referido al conocimiento sobre uno mismo como aprendiz y los factores que influyen en el desempeño; b) un conocimiento procedimental, relacionado al conocimiento sobre estrategias y otros procedimientos apropiados para resolver el problema o mejorar el aprendizaje; y c) un conocimiento condicional, alusivo al conocimiento de por qué y cuándo usar una estrategia en particular (SCHRAW; DENNISON, 1994).

3- El hecho de que uno sepa cómo funciona el cerebro no necesariamente resulta en un monitoreo y control de la cognición. 


\section{Aspectos metodológicos}

Como se señaló anteriormente, el objetivo de la investigación es configurar un marco conceptual actualizado, que dé cuenta de las propuestas teóricas del concepto, los componentes y los términos afines a la metacognición. Por esto, la investigación consistió en revisar, principalmente, las bases de datos Scopus, WoS y SCielo posteriores al año 2000. Igualmente, se inspeccionaron artículos clave en la investigación sobre la temática. En cuanto al proceso de revisión, esta se llevó a cabo entre los años 2016 y principios del año 2018. Las palabras clave que se incluyeron en la búsqueda fueron: metacognición, teorias sobre metacognición y componentes metacognitivos (tanto en inglés como en español).

En cuanto a los criterios de inclusión inicial de los artículos para la revisión, se consideró la cantidad de citaciones de los documentos (de mayor a menor) y los cuartiles de las revistas en donde fueron publicados a partir de SCImago Research Group. Otro criterio correspondió al área de investigación, ya que se escogieron, principalmente, artículos de las áreas de psicología social, psicología cognitiva, educación y afines, excluyendo temáticas relacionadas a la psiquiatría, neurología, inteligencia artificial, comportamiento animal, medicina, entre otros. De este modo, se seleccionaron alrededor de noventa artículos de un total de $250^{4}$.

Luego de la selección y revisión de los artículos, se procedió a identificar las propuestas conceptuales sobre la metacognición y sobre los componentes, subcomponentes y términos afines, de una o más investigaciones, con el fin de clarificar, de cierto modo, los conceptos que se presentaron.

\section{Revisión de la literatura}

\section{Conceptualización de la metacognición}

Durante las últimas dos décadas, diversas disciplinas han aportado a la investigación sobre metacognición. Por ejemplo, en lingüística y psicolingüística, la metacognición ha sido estudiada como factor promotor de los procesos de comprensión y producción de textos (CROMLEY; AZEVED0, 2006; GRUPO DIDACTEXT, 2015; LACON DE DE LUCIA; ORTEGA DE HOCEVAR, 2008; MINGUELA; SOLÉ; PIESCHL, 2015; PERONARD, 2002b, 2009; SILVA, 2005; VAN KRAAYENOORD, 2010; VEENMAN, 2016); en psicología cognitiva, los estudios están dirigidos en relación a la divagación mental, la teoría de la mente, la creatividad, el aprendizaje y los juicios (COSMELLI; PREISS, 2014; MIELE; MOLDEN; GARDNER, 2009; MOURGUES; PREISS; GRIGORENKO, 2014; PREISS et al., 2016; XU; METCALFE, 2016; ZALLA et al., 2015); en psicología social, se ha relacionado a los procesos de autorregulación, gestión del error e igualmente a los juicios metacognitivos (DINSMORE et al., 2015; FINN; METCALFE, 2010; METCALFE, 2009; METCALFE; FINN,

4- La búsqueda en Scopus arrojó 3554 documentos utilizando como criterio de búsqueda el concepto metacognition, incluidos los criterios de selección que se nombraron. 
2012; PINO-PASTERNAK; WHITEBREAD, 2010); y en educación, al aprendizaje autónomo en contextos escolares y desarrollo de competencias profesionales (MATEOS, 2002; OSSES; JARAMILLO, 2008; SOODLA; JÕGI; KIKAS, 2017; SPRUCE; BOL, 2015; TANNER, 2012; ZEPEDA et al., 2015), entre otras múltiples áreas.

De este modo, estas miradas, si bien, por un lado, enriquecen conceptualmente el término, por otro lado, mellan la claridad que se requiere en la investigación científica. En ese sentido, Dinsmore y otros autores (2015) encontraron que en variados estudios los investigadores no proporcionaron una definición explícita de los procesos que se estudian, dificultando la propia comprensión de estos. En la misma línea, Schunk (2008) y Kaplan (2008) investigando las diferencias entre metacognición, autorregulación y estrategias de autorregulación constataron la tendencia de los investigadores a usar términos técnicos sin definirlos, aparentemente asumiendo que los lectores ya conocían las definiciones, lo cual, en algunas disciplinas, puede convertirse en elemento de confusión, sobre todo en campos investigativos de relativa novedad.

Así, a pesar de la cuantiosa literatura y de la contundente evidencia en cuanto al funcionamiento de la metacognición, persiste cierto recelo sobre el uso del concepto, principalmente por la idea de que un sinnúmero de otros conceptos pueden ser alojados bajo la idea de metacognición, haciéndolo aún más ambiguo (IRWIN, 2017; KAPLAN, 2008). Por ello, la necesidad de una revisión teórica sobre las propuestas conceptuales (Tabla 1), ya que a partir de estas diferencias es posible identificar elementos comunes que pudiesen ayudar a clarificar el concepto.

En las definiciones, si bien hay diferencias entre las propuestas conceptuales, todas mantienen la idea de regulación de la cognición como acción que permite evaluar el funcionamiento de una determinada tarea u objetivo. En cuanto a las diferencias, estas se producen, principalmente, por la aplicabilidad que se le da a este proceso, ya que por un lado, se plantea como estrategia para lograr determinado fin (FLEMING; DOLAN, 2014; MOSTACERO, 2013; PERONARD, 2009) y por otro, sería una habilidad que en ocasiones pudiese estar automatizada (SHEA et al., 2014). En otros matices, también hay una propuesta conceptual que incorpara la precisión de reconocer no solo el propio conocimiento sino también las emociones propias y del otro (IRWIN, 2017).

\section{Componentes, subcomponentes y términos afines de la metacognición}

Los componentes que forman parte de un concepto relativamente novedoso, sea cual fuese la disciplina, van teniendo modificaciones, incorporando nuevos componentes o atributos, o tal vez, excluyendo ciertos aspectos accesorios. En cuanto a la metacognición, desde la irrupción del concepto, se reconocen dos componentes principales hasta el día de hoy: conocimiento de la cognición y regulación de la cognición. Sobre el primero, se señala que corresponde a la información acerca de una propiedad de un proceso cognitivo e incluye el conocimiento sobre uno mismo como aprendiz y los factores que pueden afectar el rendimiento, el conocimiento sobre las estrategias, y el conocimiento sobre cuándo y por qué usar determinadas estrategias (KUHN; DEAN JR., 2004; SHEA et al., 2014; VEENMAN; VAN HOUT-WOLTERS; AFFLERBACH, 2006; YEUNG; SUMMERFIELD, 2012). La regulación 
Tabla 1. Autores y propuestas teóricas sobre metacognición

\begin{tabular}{|c|c|}
\hline Autor(es) & Propuesta teórica \\
\hline $\begin{array}{l}\text { National Reading } \\
\text { Panel (2000) }\end{array}$ & $\begin{array}{l}\text { La metacognición incluida en los procesos de autorregulación tiene la capacidad de orquestar el aprendizaje: los errores de } \\
\text { planificación, monitorear el éxito, y corregir cuando sea necesario un aprendizaje intencional y efectivo [...] metacognición } \\
\text { también se refiere a la capacidad de reflexionar sobre la propia actuación. }\end{array}$ \\
\hline $\begin{array}{l}\text { Veenman, Van } \\
\text { Hout-Wolters, } \\
\text { Afflerbach (2006) }\end{array}$ & $\begin{array}{l}\text { "La metacognición se refiere al conocimiento descriptivo y al control regulador sobre el propio sistema cognitivo". (2006, } \\
\text { p. 04). }\end{array}$ \\
\hline $\begin{array}{l}\text { Schraw, Crippen y } \\
\text { Hartley (2006) }\end{array}$ & $\begin{array}{l}\text { "La metacognición se refiere a la capacidad de reflexionar, entender y controlar los propios procesos cognitivos". (2006, p. } \\
\text { 114). }\end{array}$ \\
\hline Schneider (2008) & $\begin{array}{l}\text { "La metacognición es una forma de control ejecutivo que implica el seguimiento [del rendimiento de los procesos cognitivos] } \\
\text { y la autorregulación." (2008, p. 114). }\end{array}$ \\
\hline Peronard (2009) & $\begin{array}{l}\text { "Las estrategias [metacognitivas] son conscientes e intencionadas, en consecuencia, sujetas a la autorreflexión y control." } \\
(2009, \text { p. 273). }\end{array}$ \\
\hline Metcalfe (2009) & $\begin{array}{l}\text { "El estudio de la metacognición -conocimiento de su propio conocimiento-, está motivado por la suposición de que, a mayor } \\
\text { metacognición, la gente podría lograr el control eficaz de su propio aprendizaje. Debido a esto, supone un enlace con el } \\
\text { control del aprendizaje, prestando mucha atención a la cuestión de si el monitoreo metacognitivo es o no exacto." (2009, } \\
\text { p. 159). }\end{array}$ \\
\hline Mostacero (2013) & $\begin{array}{l}\text { "Metacognición es un término de origen psicológico que designa una serie de operaciones, actividades y procedimientos que } \\
\text { operan sobre la cognición, esto es, lo que acompaña o sigue a la cognición. Se utiliza de manera espontánea en la vida cotidiana, } \\
\text { no obstante, de ella se obtienen buenos resultados en las disciplinas, sobre todo, cuando se trabaja con tareas complejas o poco } \\
\text { conocidas." (2013, p. 175). }\end{array}$ \\
\hline $\begin{array}{l}\text { McCluskey y otros } \\
\text { autores (2013) }\end{array}$ & $\begin{array}{l}\text { "Es la toma de conciencia respecto de los propios procesos de aprendizaje, de las fortalezas y debilidades que se tienen en } \\
\text { el momento de la resolución de un problema o la realización de una tarea, y respecto de la evaluación de estos procesos. La } \\
\text { metacognición hace referencia a la supervisión, evaluación, regulación y organización de los procesos que tienen relación } \\
\text { con la solución de problemas o el cumplimiento de una tarea." (2013, p. 102). }\end{array}$ \\
\hline $\begin{array}{l}\text { Mevarech y } \\
\text { Kramarski (2014) }\end{array}$ & $\begin{array}{l}\text { "La metacognición es una forma de cognición, un proceso de segundo orden de pensamiento superior que implica un control } \\
\text { activo sobre los procesos cognitivos. Permite a los estudiantes planificar y asignar recursos de aprendizaje, supervisar sus } \\
\text { conocimientos actuales y niveles de habilidad, y evaluar su nivel de aprendizaje en varios puntos durante la resolución de }\end{array}$ \\
\hline $\begin{array}{l}\text { Fleming y Dolan } \\
\text { (2014) }\end{array}$ & $\begin{array}{l}\text { "Un modelo metacognitivo es desarrollado para dar cuenta de las disociaciones de comportamiento entre el objeto nivel- } \\
\text { cognición, o más correctamente, el rendimiento y la tarea del nivel "meta", conceptualizado como el seguimiento y el control } \\
\text { del nivel de objeto." (2014, p. 1339). }\end{array}$ \\
\hline $\begin{array}{l}\text { Shea y otros } \\
\text { autores (2014) }\end{array}$ & $\begin{array}{l}\text { "Definimos la metacognición más restringida, como procesos de control que hacen uso de una o más representaciones } \\
\text { metacognitivas, es decir, representaciones de una propiedad de un proceso cognitivo. [...] La metacognición se divide en dos } \\
\text { sistemas: en el sistema } 1 \text { o metacognición implícita, se describen las propiedades de los procesos cognitivos (por ejemplo, su } \\
\text { velocidad o fiabilidad) en lugar de propiedades del mundo (por ejemplo, la ubicación o el valor de un objeto). El sistema } 2 \text { o } \\
\text { metacognición explícita, que representa propiedades de los procesos cognitivos en una forma consciente, notificable." (2014, } \\
\text { p. 186). }\end{array}$ \\
\hline $\begin{array}{l}\text { Winne y Azevedo, } \\
(2015)\end{array}$ & $\begin{array}{l}\text { "La metacognición es la cognición, donde se presenta la información sobre la que opera un alumno, describiendo sus características } \\
\text { cognitivas. La metacognición puede ocurrir antes del evento cognitivo [...] Puede ocurrir de manera simultánea con el evento, } \\
\text { cuando un alumno monitorea el progreso y considera si las tácticas y estrategias que se aplican en la actualidad cognitiva podrían } \\
\text { adaptarse o reemplazarse para mejorar el progreso. Y la metacognición puede ocurrir después del evento, cuando un alumno } \\
\text { considera retrospectivamente un evento de aprendizaje, evalúa qué tan efectiva fue, y se dedica a la transferencia y al tomar } \\
\text { decisiones sobre cómo abordar tareas similares en el futuro." (2015, p. 63). }\end{array}$ \\
\hline $\begin{array}{l}\text { James y otros } \\
\text { autores (2016) }\end{array}$ & $\begin{array}{l}\text { "La metacognición se refiere al espectro de actividades mentales que implican una reflexión sobre uno de los estados } \\
\text { mentales del pensamiento, que van desde dar cuenta o reflectar sobre un fenómeno psicológico discreto a uno cada vez } \\
\text { más complejo [...] La metacognición se diferencia de la cognición social en que la cognición social a menudo se refiere a la } \\
\text { prec yo y de los juicios sociales de uno, mientras que la metacognición hace referencia al grado de integración de un sentido }\end{array}$ \\
\hline $\begin{array}{l}\text { Aguirre Seura } \\
(2016)\end{array}$ & $\begin{array}{l}\text { "Se trata de un atributo del pensamiento humano que se relaciona con la habilidad que tienen las personas para: conocer } \\
\text { lo que se conoce, planificar estrategias para conocer, poseer conciencia de los propios pensamientos durante el acto del } \\
\text { conocimiento y, reflexionar y evaluar la productividad de su propio deseo de conocimiento." (2016, p. 184). }\end{array}$ \\
\hline Irwin (2017) & $\begin{array}{l}\text { "La metacognición es la capacidad de reconocer con precisión el propio conocimiento y emociones, así como el conocimiento y las } \\
\text { emociones de otros, la capacidad de comprender la interacción potencial o real de estos pensamientos y emociones y su impacto } \\
\text { en el comportamiento y la capacidad para identificar estos fenómenos como representaciones subjetivas que son distintas de la } \\
\text { realidad." (2017, p. 454). }\end{array}$ \\
\hline
\end{tabular}

Fuente: Elaboración propia. 
de la cognición, en cambio, corresponde a mecanismos cognitivos responsables de guiar el pensamiento y la conducta de conformidad con los objetivos e intenciones a través de actividades de planificación, conocimiento de la comprensión y desempeño de tareas, y evaluación de la eficacia de los procesos y del uso de las estrategias de monitoreo (SHEA et al., 2014; THOMAS; BARKSDALE-LADD, 2000; VEENMAN, 2016).

Sin embargo, a pesar de los componentes anteriores, la investigación ha propuesto otros mecanismos vinculados a ciertos procesos. Por ejemplo, los juicios metacognitivos, que corresponden a las estimaciones sobre aciertos y desaciertos de acuerdo con un objetivo; el cual a su vez, es especificado en otros tipos de juicios dependiendo del momento de realización de este o de lo que se evalúa, como los juicios de dificultad (judgments of difficulty - JOD), los juicios de aprendizaje (judgments of learning - JOL), las sensaciones de saber (feeling of knowing - FOK), los juicios retrospectivos de confianza (retrospective confidence judgments - RCJ) o las predicciones de rendimiento (predictions of performance - POP). Todos ellos relacionados a la idea monitorear y evaluar antes, durante, o después, la actividad cognitiva realizada.

A continuación, se presentan diferentes conceptos que la investigación revisada vincula, de cierta manera, a la metacognición (Tabla 2). En ella, se encuentran términos más afınes al proceso metacognitivo, por ejemplo, calibración o fluidez; o términos que la investigación ha emparentado a este proceso, como es el de aprendizaje autorregulado o el de funciones ejecutivas.

Tabla 2. Componentes, subcomponentes y términos afines de la metacognición

\begin{tabular}{|c|c|}
\hline Concepto & Propuesta teórica* \\
\hline Autorregulación & $\begin{array}{l}\text { Se refiere a la vigilancia y control de todos los aspectos del funcionamiento humano, incluyendo el plano } \\
\text { emocional, social y aspectos motivacionales (PANADERO; ALONSO-TAPIA, 2014; PINO-PASTERNAK; } \\
\text { WHITEBREAD, 2010; WHITEBREAD; PINO PASTERNAK, 2010). }\end{array}$ \\
\hline $\begin{array}{l}\text { Aprendizaje autorregulado } \\
\text { Self-Regulated Learning (SRL) }\end{array}$ & $\begin{array}{l}\text { Es un proceso activo y constructivo mediante el cual los estudiantes fijan metas para su aprendizaje, que tratan } \\
\text { de controlar y regular, su cognición, la motivación y el comportamiento, guiado y limitado por sus objetivos y las } \\
\text { características del contexto (AZEVEDO, 2009; PINTRICH, 2000; WHITEBREAD; PINO PASTERNAK, 2010). }\end{array}$ \\
\hline Calibración & $\begin{array}{l}\text { La calibración se determina haciendo coincidir los juicios con el desempeño real en una tarea de criterio; los juicios mejor } \\
\text { pareados se consideran más precisos o bien calibrados y se supone que reflejan un mejor monitoreo (hay que tener } \\
\text { en cuenta que una buena monitorización también puede ocurrir con una comprensión baja). Un resultado pobre en la } \\
\text { calibración demuestra que los estudiantes tienden a sobreestimar su comprensión (MINGUELA; SOLÉ; PIESCHL, 2015). }\end{array}$ \\
\hline Capacidad metacognitiva & $\begin{array}{l}\text { Capacidad (individual) para asignar una menor confianza a sus errores (HAINGUERLOT; VERGNAUD; DE } \\
\text { GARDELLE, 2018). }\end{array}$ \\
\hline Conducta metacognitiva & $\begin{array}{l}\text { Corresponde al comportamiento metacognitivo, en relación a las frecuencias de cambio de los sujetos en el uso } \\
\text { de la estrategia, el cual puede ser adaptativo o estático (BINBASARAN TUYSUZOGLU; GREENE, 2015). }\end{array}$ \\
\hline Consciencia (awareness) & $\begin{array}{l}\text { La metacognición tiene menos procesamiento consciente por naturaleza, por ejemplo, si las ideas acerca de sí } \\
\text { mismo han sido firmemente establecidas o si la actividad de control de sí mismo se ha convertido en un regulador } \\
\text { de un buen hábito. Entonces en el auto-monitoreo y evaluación de los procesos que se ejecutan en el fondo } \\
\text { (background) de los procesos cognitivos que están siendo ejecutados, se detecta un error, justificadamente o no, } \\
\text { en el sistema pasa a ser alertado (VEENMAN; VAN HOUT-WOLTERS; AFFLERBACH, 2006). }\end{array}$ \\
\hline Control metacognitivo & $\begin{array}{l}\text { El control metacognitivo forma parte del proceso de regulación y podría surgir como decisión del monitoreo } \\
\text { (DINSMORE et al., 2015; SCHWONKE et al., 2013; SON, 2010). }\end{array}$ \\
\hline \multirow{2}{*}{ Efecto Dunning -Kruger } & $\begin{array}{l}\text { Se refiere a que mientras más incompetente es una persona, menos nota su incompetencia, y por el contrario, } \\
\text { mientras más competente, más subvalora su competencia (KRUGER; DUNNING, 1999). }\end{array}$ \\
\hline & $\begin{array}{l}\text { Corresponde a la incongruencia en las predicciones de desempeño que realizan sujetos con bajos niveles de } \\
\text { competencia, las cuales por lo general, no están en línea con su desempeño (DUNNING et al., 2003). }\end{array}$ \\
\hline
\end{tabular}




\begin{tabular}{|c|c|}
\hline Estrategia & $\begin{array}{l}\text { La estrategia es un proceso cognitivo y metacognitivo específico que busca la consecución de objetivos, a } \\
\text { través de una planificación consciente e intencionada (GRUPO DIDACTEXT, 2015). }\end{array}$ \\
\hline \multirow{2}{*}{ Estrategias cognitivas } & Corresponde a las estrategias que están relacionadas con el logro de las metas (GASKINS; THORNE, 1999). \\
\hline & Las estrategias cognitivas se refieren a las habilidades necesarias para realizar una tarea (ESCORCIA et al., 2017). \\
\hline \multirow{4}{*}{ Estrategias metacognitivas } & Son las estrategias que controlan el proceso en sus avances, interferencias o retrocesos (GASKINS; THORNE, 1999). \\
\hline & Las estrategias metacognitivas se necesitan para entender cómo se realiza una tarea (ESCORCIA et al., 2017). \\
\hline & $\begin{array}{l}\text { Conjunto de acciones orientadas a conocer las propias operaciones y procesos mentales (qué), saber utilizarlas (cómo) } \\
\text { y saber readaptarlas y/o cambiarlas cuando así lo requieran las metas propuestas (OSSES; JARAMILLO, 2008). }\end{array}$ \\
\hline & $\begin{array}{l}\text { Las estrategias metacognitivas preparan a los alumnos para tener el control de las variables: tarea, persona, estrategia } \\
\text { y ambiente (GRUPO DIDACTEXT, 2015). }\end{array}$ \\
\hline Experiencia metacognitiva & $\begin{array}{l}\text { Se refiere a los procesos conscientes o inconscientes que acompañan cualquier éxito o fracaso en el aprendizaje o la } \\
\text { realización de una empresa cognitiva (MEVARECH; KRAMARSKI, 2014). }\end{array}$ \\
\hline Funciones ejecutivas & $\begin{array}{l}\text { Son una variedad de procesos cognitivos que se requieren para alcanzar un objetivo. Esta incluye memoria de trabajo, } \\
\text { control inhibitorio, atención, y control y cambio de atención (IRWIN, 2017; LUTHER et al., 2016). }\end{array}$ \\
\hline Habilidades metacognitivas & $\begin{array}{l}\text { La diferencia esencial entre una estrategia y una habilidad es que las estrategias requieren un esfuerzo deliberado } \\
\text { y consciente, mientras que la ejecución de las habilidades es (en parte) automatizada (tal como ocurre con las } \\
\text { habilidades metacognitivas) (AFFLERBACH; PEARSON; PARIS, 2008; VEENMAN, 2016). }\end{array}$ \\
\hline Juicios de dificultad (JOD) & $\begin{array}{l}\text { Es una estrategia de metacomprensión y corresponde al monitoreo de la comprensión juzgando la dificultad que se } \\
\text { siente entender un texto en la medida en que se lee (OZURU; KURBY; MCNAMARA, 2012). }\end{array}$ \\
\hline $\begin{array}{l}\text { Juicios de facilidad de } \\
\text { aprendizaje o Ease-of-learning } \\
\text { Judgments (EOL) }\end{array}$ & $\begin{array}{l}\text { Corresponde a un tipo de vigilancia prospectiva, cuyo fin es predecir lo fácil que será aprender (MIHALCA; } \\
\text { MENGELKAMP; SCHNOTZ, 2017; VAN LOON et al., 2015). }\end{array}$ \\
\hline \multirow{2}{*}{$\begin{array}{l}\text { Juicios o sentencias de } \\
\text { aprendizaje o judgments of } \\
\text { learning (JOL) }\end{array}$} & $\begin{array}{l}\text { Estimaciones de lo bien o mal que han aprendido algo las personas de acuerdo a su objetivo de aprendizaje deseado, } \\
\text { generando juicios subjetivos sobre el rendimiento actual. Estos juicios pueden ser positivos o negativos (BINBASARAN } \\
\text { TUYSUZOGLU; GREENE, 2015). }\end{array}$ \\
\hline & Corresponden a los juicios sobre el propio desempeño (SCHLEINSCHOK; EITEL; SCHEITER, 2017). \\
\hline $\begin{array}{l}\text { Juicios retrospectivos de } \\
\text { confianza o retrospective } \\
\text { confidence judgments (RCJ) }\end{array}$ & $\begin{array}{l}\text { Es el monitoreo que se realiza a posteriori de pedir a los estudiantes que evaluaran su confianza sobre haber } \\
\text { aprendido con éxito. La exactitud de estos juicios metacognitivos se estimó en términos de la diferencia entre el } \\
\text { rendimiento juzgado y el real (es decir, la exactitud absoluta o de calibración) (DINSMORE et al., 2015; HAINGUERLOT; } \\
\text { VERGNAUD; DE GARDELLE, 2018; MHALCA; MENGELKAMP; SCHNOTZ, 2017). }\end{array}$ \\
\hline $\begin{array}{l}\text { Metas cognitivas (cognitive } \\
\text { goa/s) }\end{array}$ & $\begin{array}{l}\text { Las metas cognitivas desencadenan el uso del conocimiento metacognitivo, que a su vez activa los otros } \\
\text { componentes metacognitivos (MEVARECH; KRAMARSKI, 2014). }\end{array}$ \\
\hline Metamemoria & $\begin{array}{l}\text { Se refiere a los procesos y estructura por el que las personas son capaces de examinar el contenido de sus memorias, } \\
\text { a futuro o de manera retroactiva, haciendo juicios o comentarios acerca de ellos (METCALFE; DUNLOSKY, 2008). }\end{array}$ \\
\hline \multirow{3}{*}{ Monitoreo metacognitivo } & $\begin{array}{l}\text { El monitoreo metacognitivo se refiere a la relación entre el desempeño de tareas y un juicio acerca del rendimiento } \\
\text { (GUTIERREZ et al., 2016). }\end{array}$ \\
\hline & $\begin{array}{l}\text { Es la capacidad de evaluar el estado de aprendizaje en relación con los objetivos propuestos [...] El monitoreo puede } \\
\text { ser medido para pedir a los sujetos dar juicios sobre su desempeño (SCHLEINSCHOK; EITEL; SCHEITER, 2017). }\end{array}$ \\
\hline & $\begin{array}{l}\text { En situaciones de lectura, la evidencia sugiere que el monitoreo es cuando un lector necesita información para } \\
\text { una decisión metacognitiva, la que se basa principalmente en la capacidad de los estudiantes para monitorear con } \\
\text { precisión su progreso de aprendizaje, y no en sus habilidades generales de comprensión (MAÑÁ; VIDAL-ABARCA; } \\
\text { SALMERÓN, 2017). }\end{array}$ \\
\hline Precursores metacognitivos & $\begin{array}{l}\text { Están relacionados con el desarrollo de las habilidades afines a la comprensión social, la comprensión emocional, el } \\
\text { lenguaje comprensivo y expresivo y el desarrollo del juego simbólico. Es decir, a algo que sucedió o existió antes que } \\
\text { otra cosa, especialmente si se desarrolló o influyó en ello ( SÁlZ; CARBONERO 2017). }\end{array}$ \\
\hline \multirow[b]{2}{*}{$\begin{array}{l}\text { Predicciones de rendimiento } \\
0 \text { desempeño (POP) }\end{array}$} & Son las predicciones de desempeño que realiza un sujeto (VAN LOON et al., 2015). \\
\hline & $\begin{array}{l}\text { Es una estrategia de metacomprensión, en donde se supervisa la comprensión tomando en cuenta lo bien que se } \\
\text { debería realizar, por ejemplo, en preguntas de pruebas futuras basadas en el texto que se está leyendo (OZURU; } \\
\text { KURBY; MCNAMARA, 2012). }\end{array}$ \\
\hline Representación metacognitiva & $\begin{array}{l}\text { Representación de una propiedad de un proceso cognitivo, por ejemplo, la fiabilidad de una representación perceptual } \\
\text { (SHEA et al., 2014). }\end{array}$ \\
\hline Sensación de saber (FOK) & $\begin{array}{l}\text { Es un juicio sobre un aspecto diferente de la memoria. Es saber la respuesta a una pregunta en particular a pesar de } \\
\text { ser incapaz de recordarla explícitamente (FLEMING; DOLAN, 2014). }\end{array}$ \\
\hline
\end{tabular}

* Las propuestas teóricas están traducidas, en su mayoría, del inglés al español. Además, están ordenadas en orden alfabético.

Fuente: Elaboración propia. 


\section{Discusión}

\section{Conceptualización}

Las investigaciones sobre metacognición sostienen que este es, en primer lugar $\mathrm{y}$ antes que todo, un proceso cognitivo que abarca principalmente la regulación de otros procesos cognitivos (FLAVELL, 1979; VEENMAN, 2016; WINNE; AZEVED0, 2015). Asimismo, la activación de la metacognición es bajo ciertas condiciones, por ejemplo, tareas complejas ${ }^{5}$, con el fin de monitorear y regular los procesos cognitivos que requiera la realización de dicha tarea (MEVARECH; KRAMARSKI, 2014; VEENMAN, 2016). En ese sentido, la investigación destaca el carácter estratégico que puede asumir, por ejemplo, en los contextos educativos. Sin embargo, es necesario aclarar que este es un proceso cuyo fin permite, en determinadas situaciones, alcanzar un objetivo. Y de acuerdo a lo revisado, no se propone como un fin en sí mismo, como a veces se quiere hacer notar (MARZANO; PICKERING, 2005).

En esa misma línea, en cuanto al carácter estratégico que asume la metacognición, la literatura distingue diferencias entre estrategias cognitivas y estrategias metacognitivas. Las estrategias cognitivas se refieren a las habilidades necesarias para realizar una tarea, en cambio, las estrategias metacognitivas se necesitan para entender cómo se realiza dicha tarea (ESCORCIA et al., 2017). En la literatura, además, se hace una diferencia en cuanto a los dominios de la metacognición, ya que por un lado, la investigación sugiere que esta tiene un carácter más general (FLEMING; LAU, 2014; OSSES; JARAMILLO, 2008), sin embargo, hay otra que señala que corresponde a un dominio más específico (CAMPO et al., 2016; SCHNEIDER; ARTELT, 2010; VEENMAN, 2016). Sobre esto último, la literatura propone a la metacognición como un factor para desarrollar aprendizaje en un determinado y acotado contexto. Por ejemplo, en el desarrollo de la escritura académica (ESCORCIA et al., 2017; GRUPO DIDACTEXT, 2015) o en la enseñanza de las matemáticas (SCHNEIDER; ARTELT, 2010; VAN DER STEL et al., 2010), o en la comprensión lectora (de hecho se habla de metacomprensión) (AFFLERBACH; CHO, 2009; PERONARD, 2002b; VEENMAN, 2016).

Otro punto en discusión en cuanto a la conceptualización corresponde a la relación entre los procesos de control cognitivo: metacognición, autorregulación y aprendizaje autorregulado. Si bien se ha considerado que hay una relación intrínseca entre estos procesos, se proponen ciertas diferencias (DINSMORE; ALEXANDER; LOUGHLIN, 2008; KAPLAN, 2008; WHITEBREAD; PINO PASTERNAK, 2010; ZEPEDA et al., 2015). Así, se señala que el primero corresponde al procesamiento cognitivo que da lugar a la regulación de otros procesos (por ejemplo, motores), en cambio, los otros dos, son procesos más amplios que conjugan otro tipo de elementos además de la cognición. Por esto se sostiene que la metacognición forma parte esencial del proceso de autorregulación (PETERSBURTON; BOTOV, 2017; PINTRICH, 2004; RICHARDSON; ABRAHAM; BOND, 2012). En ese

5- Se definen como tareas complejas la lectura o la escritura (VEENMAN, 2016). 
sentido, si se aboga por el desarrollo de la autonomía y la autorregulación, por ejemplo, en contextos escolares, la enseñanza de estrategias metacognitivas es fundamental.

\section{Componentes, subcomponentes y términos afines}

En la investigación sobre metacognición destaca, en primer lugar, el énfasis que se le da a la regulación de la cognición. Por ejemplo, en los últimos 20 artículos de la revista Metacognition and learning del año 2017, al menos 15 de estos artículos, abordan de manera explícita esta temática. No obstante, esta especificidad en un componente pareciera ser solo algo baladí, ya que la metacognición per se integra tanto un conocimiento de la cognición como una regulación de la cognición, lo cual no implica que conocer el funcionamiento cognitivo o del uso de estrategias, sea sinónimo de regulación cognitiva (BROWN, 1987; GUTIERREZ et al., 2016). A pesar de ello, destaca el énfasis que se le da a una por sobre la otra.

Otro de los puntos fundamentales sobre los componentes de la metacognición, es la relativa confusión en cuanto a la diferencia entre regulación, monitoreo y control metacognitivo. Sobre el primero, cabe señalar que corresponde a uno de los dos componentes principales en el modelo metacognitivo, en el cual se insertan los conceptos de monitoreo y control. En cambio, el monitoreo metacognitivo, como recién se estableció, es parte del proceso de regulación e implica el seguimiento del procesamiento cognitivo. Por último, el control metacognitivo, también forma parte del proceso de regulación, sin embargo, este surge como decisión del monitoreo metacognitivo (NELSON; NARENS, 1994; SCHWONKE et al., 2013; STERNBERG, 2009; WINNE; AZEVEDO, 2015; YEUNG; SUMMERFIELD, 2012).

Otra idea que tiene una amplia presencia en la literatura corresponde a los juicios metacognitivos (METCALFE, 2009; MUELLER; DUNLOSKY, 2017; NEGRETTI, 2015; PIEGER; MENGELKAMP; BANNERT, 2016). Este concepto es uno de los elementos que ha permitido operacionalizar la idea de metacognición. Pese a ello, la especificidad que le han dado algunos autores hace que pierda, en ocasiones, el carácter operativo. En ese sentido, la propuesta de juicios metacognitivos viene a responder uno de los desafíos que señalaba Schunk (2008) en cuanto a las mediciones sobre el control cognitivo. Así, la evaluación de la precisión de estos juicios se convierte en un punto clave para la toma de decisiones en consideración a las tareas y el rendimiento en ellas, los cuales, a partir de las claves conductuales, permitiría establecer declaraciones no solo correlacionales sino también de tipo causal (MANISCALCO; LAU, 2012).

\section{Conclusión}

El objetivo de la presente revisión ha sido identificar las propuestas teóricas que la literatura especializada señala sobre la metacognición, sus componentes, subcomponentes y términos afines. Por ello, se realizó un recorrido histórico del concepto, con el fin de facilitar la comprensión de las propuestas actuales a partir de la literatura especializada inicial. 
Se expuso que la metacognición es un concepto de la psicología cognitiva que se centra en la participación del individuo en su proceso de pensamiento. Por esto, el concepto se vuelve relevante, principalmente en las situaciones de aprendizaje. Así, esta importancia en el campo de la educación, se debe al supuesto de que enfatizar en el uso de estrategias metacognitivas, se antepone a la idea de aprendizaje incidental, ya que un aprendiz experto o competente, es un participante intencional y activo, es decir, estratégico y consciente (ALAMARGOT et al., 2011). Sin embargo, a pesar de esto y de la evidencia encontrada, persisten dudas sobre su funcionamiento en contextos de aprendizaje. Por ejemplo, aún no queda claro si la metacognición forma parte del aprendizaje como dominio general o como dominio específico de alguna disciplina. También se cuestiona la precisión de los juicios metacognitivos y la relación de estos con el efecto DunningKruger. A pesar de ello, la literatura es consistente en cuanto a evidencia sobre el impacto que tiene la metacognición en el aprendizaje: se estima que la habilidad metacognitiva en algunos estudios representó el 40\% de los resultados de aprendizaje (RICHARDSON; ABRAHAM; BOND, 2012; VEENMAN, 2008, 2016).

Por último, cabe señalar que, si bien la metacognición es, sin lugar a duda, un campo de estudio de singular valor en el ámbito de la psicología, de la pedagogía, de la lingüística, entre otras múltiples disciplinas, todavía existen otras interrogantes por dilucidar. Por ejemplo, medir cuál es el impacto de la enseñanza de estrategias metacognitivas en relación a la regulación de la divagación mental (mind wandering) y cómo afectaría esto los procesos de creatividad (por ejemplo, clases de literatura); cuál es el rol de la memoria de trabajo en el proceso metacognitivo, sobre todo en sujetos competentes, vale decir, con alto grado de automatización de los procesos; cuál es el impacto de las propuestas didácticas o estrategias de enseñanza centradas en explicitar lo metacognitivo en la memoria de trabajo; qué mecanismos conductuales permiten evaluar la actividad metacognitiva en situaciones como lectura o escritura; entre muchas otras interrogantes.

\section{Referencias}

AFFLERBACH, Peter; $\mathrm{CHO}$, Byeong-Young. Identifying and describing constructively responsive comprehension strategies in new and traditional forms of reading. In: ISRAEL, Susan; DUFFY, Gerald (Ed.). Handbook of research on reading comprehension. New York: Routledge, 2009. p. 69-90.

AFFLERBACH, Peter; PEARSON, David; PARIS, Scoott. Clarifying differences between reading skills and reading strategies. The Reading Teacher, Nueva Jersey, v. 61, n. 5, p. 364-373, 2008.

AGUIRRE SEURA, Luis. Evaluación de una propuesta para el desarrollo de la escritura en estudiantes universitarios a partir de habilidades de metacognición. Logos, La Serena, v. 26, n. 2, p. 181-196, 2016.

ALAMARGOT, Denis et al. What makes a skilled writer ? Working memory and audience awareness during text composition. Learning and Individual Differences, Amsterdam, v. 21, n. 5, p. 505-516, 2011.

AZEVED0, Roger. Theoretical, conceptual, methodological, and instructional issues in research on metacognition and self-regulated learning: a discussion. Metacognition and Learning, New York, v. 4, n. 1, p. 87-95, 2009. 
BINBASARAN TUYSUZOGLU, Banu; GREENE, Jeffrey Alan. An investigation of the role of contingent metacognitive behavior in self-regulated learning. Metacognition and Learning, New York, v. 10, n. 1, p. 77-98, 2015.

BROWN, Ann. Metacognition, executive control, self-regulation and other more mysterious mechanisms. In: WEINERT, Franz; KLUWE, Rainer (Ed.). Metacognition, motivation and understanding. Hillsdale: L. Erlbaum, 1987. p. 65-116.

BROWN, Ann. et al. Learning, remembering and understanding: technical report. center for the study of reading. Champaign: Center for the Study of Reading, 1982.

CAMPO, Kiara et al. Metacognición , escritura y rendimiento académico en universitarios de Colombia y Francia. Avances en Psicología Latinoamericana, Bogotá, v. 34, n. 2, p. 233-252, 2016.

COSMELLI, Diego; PREISS, David. On the temporality of creative insight: a psychological and phenomenological perspective. Frontiers in Psychology, Lausanne, v. 5, article 1184, p. 1-6, 2014.

CROMLEY, Jennifer; AZEVEDO, Roger. Self-report of reading comprehension strategies: what are we measuring? Metacognition and Learning, New York, v. 1, n. 3, p. 229-247, 2006.

DINSMORE, Daniel; ALEXANDER, Patricia; LOUGHLIN, Sandra. Focusing the conceptual lens on metacognition, self-regulation, and self-regulated learning. Educational Psychology Review, New York, v. 20, n. 4, p. 391-409, dic. 2008.

DINSMORE, Daniel et al. The effects of persuasive and expository text on metacognitive monitoring and control. Learning and Individual Differences, Amsterdam, v. 38, p. 54-60, 2015.

DUNNING, David et al. Why people fail to recognize their own incompetence. Current Directions in Psychological Science, Washington, DC, v. 12, n. 3, p. 83-87, 2003.

ESCORCIA, Dyanne; FENOUILLET, Fabien. Quel rôle de la métacognition dans les performances en écriture ? Analyse de la situation d 'étudiants en sciences humaines et sociales. Revue Canadienne de L'Éducation, Ottawa, v. 34, n. 2, p. 53-76, 2011.

ESCORCIA, Dyanne et al. Profiling writers: analysis of writing dynamics among college students. Metacognition and Learning, New York, v. 12, n. 2, p. 233-273, 2017.

FINN, Bridgit; METCALFE, Janet. Scaffolding feedback to maximize long-term error correction. Memory \& Cognition, Madison, v. 38, n. 7, p. 951-961, 2010.

FLAVELL, John. Desarrollo cognitivo: pasado, presente y futuro. Developmental Psychology, Washington, DC, v. 28, n. 6, p. 998-1005, 1992.

FLAVELL, John. Metacognition and cognitive monitoring: a new area of cognitive-developmental inquiry. American Psychologist, Washington, DC, v. 34, n. 10, p. 906-911, 1979.

FLAVELL, John. Metacognitive aspects of problem solving. In: RESNICK, Lauren (Ed.). The nature of intelligence. Hillsdale: Lawrence Erlbaum, 1976. p. 231-236. 
FLEMING, Stephen; DOLAN, Raymond. Philosophical Transactions of the Royal Society B: Biological Sciences, Londres, v. 367, n. 1594, p. 1338-1349, 2014.

FLEMING, Stephen; LAU, Hakwan. How to measure metacognition. Frontiers in Human Neuroscience, Lausanne, v. 8, n. 443, p. 1-9, jul. 2014.

GALLEGOS, Miguel et al. Las nuevas dinámicas del conocimiento científico y su impacto en la Psicología Latinoamericana. Individuo y Sociedad, Madrid, v. 13, n. 3, p. 106-117, 2014.

GARAY, Cristian; KEEGAN, Eduardo. Terapia metacognitiva: el síndrome cognitivo atencional y los procesos cognitivos. Revista Argentina de Clínica Psicológica, Buenos Aires, v. 25, n. 2, p. 125-134, 2016.

GASKINS, Irene; THORNE, Elliot. Cómo enseñar estrategias cognitivas en la escuela. Buenos Aires: Paidós Ibérica, 1999.

GRUPO DIDACTEXT. Nuevo marco para la producción de textos académicos. Didáctica, Lengua y Literatura, Madrid, v. 27, p. 219-254, 2015.

GUTIERREZ, Antonio et al. A two-process model of metacognitive monitoring: evidence for general accuracy and error factors. Learning and Instruction, Lovaina, v. 44, p. 1-10, 2016.

HAINGUERLOT, Marine; VERGNAUD, Jean Christophe; DE GARDELLE, Vincent. Metacognitive ability predicts learning cue-stimulus associations in the absence of external feedback. Nature, London, v. 8, n. 5602, p. 1-8, abr. 2018.

HAMM, Jay; LYSAKER, Paul. Psychoanalytic phenomenology of schizophrenia: synthetic metacognition as a construct for guiding investigation. Psychoanalytic Psychology, Washington, DC, v. 33, n. 1, p. 147-160, 2016.

HEYES, Cecilia. Who knows? Metacognitive social learning strategies. Trends in Cognitive Sciences, Cambridge, v. 20, n. 3, p. 204-213, 2016.

IRWIN, Lindsey. Metacognition: a concept analysis. Archives of Psychiatric Nursing, New York, v. 31, n. 5, p. 454-456, 2017.

JAMES, Alison et al. Metacognition moderates the relationship between dysfunctional self-appraisal and social functioning in prolonged schizophrenia independent of psychopathology. Comprehensive Psychiatry, New York, v. 69, p. 62-70, 2016.

KAPLAN, Avi. Clarifying metacognition, self-regulation, and self-regulated learning: what's the purpose? Educational Psychology Review, New York, v. 20, n. 4, p. 477-484, 2018.

KELLY, Karen; METCALFE, Janet. Metacognition of emotional face recognition. Emotion, Washington, DC, v. 11, n. 4, p. 896-906, 2011.

KORIAT, Asher. The relationships between monitoring, regulation and performance. Learning and Instruction, Lovaina, v. 22, n. 4, p. 296-298, 2012. 
KRUGER, Justin; DUNNING, David. Unskilled and unaware of it: how difficulties in recognizing one's own incompetence lead to inflated self-assessments. Journal of Personality and Social Psychology, Washington, DC, v. 77, n. 6, p. 1121-1134, 1999.

KUHN, Deanna; DEAN JR., David. Metacognition: a bridge between cognitive psychology and educational practice. Theory Into Practice, London, v. 43, n. 4, p. 268-273, 2004

LACON DE DE LUCIA, Nelsi; ORTEGA DE HOCEVAR, Susana. Cognición, metacognición y escritura. Revista Signos, Valparaíso, v. 41, n. 67, p. 231-255, 2008.

LUEDDEKE, Sara; HIGHAM, Philip. Expertise and gambling: using type 2 signal detection theory to investigate differences between regular gamblers and nongamblers. Quarterly Journal of Experimental Psychology, London, v. 64, n. 9, p. 1850-1871, 2011.

LUTHER, Lauren et al. Intrinsic motivation as a mediator between metacognition deficits and impaired functioning in psychosis. British Journal of Clinical Psychology, London, v. 55, n. 3, p. 332-347, 2016.

MAÑÁ, Amelia; VIDAL-ABARCA, Eduardo; SALMERÓN, Ladislao. Effect of delay on search decisions in a task-oriented reading environment. Metacognition and Learning, New York, v. 12, n. 1, p. 113-130, 2017.

MANISCALCO, Brian; LAU, Hakwan. A signal detection theoretic approach for estimating metacognitive sensitivity from confidence ratings. Consciousness and Cognition, San Diego, v. 21, n. 1, p. 422-430, 2012.

MARTÍ, Eduardo. Metacognición: Entre la fascinación y el desencanto. Infancia y Aprendizaje, London, n. 72 , p. 9-32, 1995.

MARZANO, Robert; PICKERING, Debra. Dimensiones del aprendizaje: manual del maestro. Tlaquepaque: Iteso, 2005.

MATEOS, Mar. Metacognición y educación. Madrid: Aique, 2002.

MAZZONI, Giuliana; SCOBORIA, Alan; HARVEY, Lucy. Nonbelieved memories. Psychological Science, Washington, DC, v. 21, n. 9, p. 1334-1340, 2010.

MCCLUSKEY, Ken et al. The amphitheater model for talent development: recognizing and nurturing the gifts of our lost prizes. International Journal for Talent Development and Creativity, Delta, v. 1, n. 1, p. 99-112, 2013.

METCALFE, Janet. Metacognitive judgments and control of study. Current Directions in Psychological Science, Washington, DC, v. 18, n. 3, p. 159-163, 2009.

METCALFE, Janet; DUNLOSKY, John. Metamemory. In: ROEDIGER, Henry (Ed.). Cognitive psychology of memory. Oxford: Elsevier, 2008. p. 349-362.

METCALFE, Janet; FINN, Bridgid. Hypercorrection of high confidence errors in children. Learning and Instruction, Lovaina,v. 22, n. 4, p. 253-261, 2012. 
MEVARECH, Zemira; KRAMARSKI, Bracha. Critical maths for innovative societies: the role of metaognitive pedagogies. Paris: OECD, 2014.

MIELE, David; MOLDEN, Daniel; GARDNER, Wendy. Motivated comprehension regulation: vigilant versus eager metacognitive control. Memory \& Cognition, Madison, v. 37, n. 6, p. 779-95, 2009.

MIHALCA, Loredana; MENGELKAMP, Christoph; SCHNOTZ, Wolfgang. Accuracy of metacognitive judgments as a moderator of learner control effectiveness in problem-solving tasks. Metacognition and Learning, New York, v. 12, p. 357-379, 2017.

MINGUELA, Marta; SOLÉ, Isabel; PIESCHL, Stephanie. Flexible self-regulated reading as a cue for deep comprehension: evidence from online and offline measures. Reading and Writing, Amsterdam, v. 28, n. 5, p. $721-744,2015$.

MOSTACERO, Rudy. Construcción de la reseña mediante estrategias metacognitivas. Lenguaje, Cali, v. 41, n. 1, p. 169-200, 2013.

MOURGUES, Catalina; PREISS, David; GRIGORENKO, Elena. Reading skills, creativity, and insight: exploring the connections. The Spanish Journal of Psychology, Madrid, v. 17, n. 58, p. 1-10, 2014.

MUELLER, Michael; DUNLOSKY, John. How beliefs can impact judgments of learning: evaluating analytic processing theory with beliefs about fluency. Journal of Memory and Language, New York, v. 93, p. 245-258, 2017.

NATIONAL READING PANEL. Teaching children to read: an evidence-based assessment of the scientific research literature on reading and its implications for reading instruction. Bethesda, MD: NRP, 2000.

NEGRETTI, Raffaella. Calibrating genre: metacognitive judgments and rhetorical effectiveness in academic writing by L2 graduate students. Applied Linguistics, London, v. 38, n. 4, p. 512-539, 2015.

NELSON, Thomas. Consciousness and metacognition. American Psychologist, Washington, DC, v. 51, n. 2 , p. 102-116, 1996.

NELSON, Thomas; NARENS, Louis. Why investigate metacognition? In: METCALFE, Janet; SHIMAMURA, Arthur P. (Ed.). Metacognition: knowing about knowing. Cambridge: MIT Press, 1994. p. 1-25.

NICKERSON, Raymond; PERKINS, David; SMITH, Edward. Enseñar a pensar: aspectos de la actitud intelectual. New Jersey: Lawrence Erlbaum, 1994.

OSSES, Sonia; JARAMILLO, Sandra. Metacognición: un camino para aprender a aprender. Estudios Pedagógicos, Valdivia, v. 34, n. 1, p. 187-197, 2008.

OZURU, Yasuhiro; KURBY, Christopher; MCNAMARA, Danielle. The effect of metacomprehension judgment task on comprehension monitoring and metacognitive accuracy. Metacognition and Learning, New York, v. 7 , n. 2, p. 113-131, 2012. 
PANADERO, Ernesto; ALONSO-TAPIA, Jesús. ¿Cómo autorregulan nuestros alumnos? Modelo de Zimmerman sobre estrategias de aprendizaje. Anales de Psicología, Murcia, v. 30, n. 2, p. 450-462, 2014.

PERONARD, Marianne. Conocimiento de estrategias de lectura y metacomprensión. Onomázein, Santiago de Chile, v. 7, p. 95-115, 2002b.

PERONARD, Marianne. Los estudios metacognitivos y sus raíces en el tiempo. Boletín de Filología, Santiago de Chile, v. 39, p. 123-140, $2002 \mathrm{a}$.

PERONARD, Marianne. Metacognición: mente y cerebro. Boletín de Filología, Santiago de Chile, v. 44, n. 2, p. 263-275, 2009.

PETERS-BURTON, Erin; BOTOV, Ivan. Self-regulated learning microanalysis as a tool to inform professional development delivery in real-time. Metacognition and Learning, New York, v. 12, n. 1, p. 45-78, 2017.

PIEGER, Elisabeth; MENGELKAMP, Christoph; BANNERT, Maria. Metacognitive judgments and disfluency: Does disfluency lead to more accurate judgments, better control, and better performance? Learning and Instruction, Lovaina, v. 44, p. 31-40, 2016.

PINO-PASTERNAK, Deborah; WHITEBREAD, David. The role of parenting in children's self-regulated learning. Educational Research Review, Lovaina, v. 5, n. 3, p. 220-242, 2010.

PINTRICH, Paul. A conceptual framework for assessing motivation and self-regulated learning in college students. Educational Psychology Review, San Diego, v. 16, n. 4, p. 385-407, 2004.

PINTRICH, Paul. The role of goal orientation in self-regulated learning. In: BOEKAERTS, Monique; ZEIDNER, Moshe; PINTRICH, Paul (Ed.). Handbook of self-regulation. San Diego: Academic Press, 2000. p. 451-502.

PREISS, David et al. Examining the influence of mind wandering and metacognition on creativity in university and vocational students. Learning and Individual Differences, Amsterdam, v. 51, p. 417-426, 2016.

RICHARDSON, Michelle; ABRAHAM, Charles; BOND, Rod. Psychological correlates of university students' academic performance: a systematic review and meta-analysis. Psychological Bulletin, Washington DC, v. 138, n. 2, p. 353-387, 2012.

SÁlZ, María Consuelo; CARBONERO, Miguel Ángel. Metacognitive precursors: an analysis in children with different disabilities. Brain Sciences, Basilea, v. 7, n. 10, 2017.

SCHLEINSCHOK, Katrin; EITEL, Alexander; SCHEITER, Katharina. Do drawing tasks improve monitoring and control during learning from text? Learning and Instruction, Lovaina, v. 51, p. 10-25, 2017.

SCHNEIDER, Wolfgang. The development of metacognitive knowledge in children and adolescents: major trends and implications for education. Mind, Brain, and Education, New Jersey, v. 2, n. 3, p. 114-121, 2008.

SCHNEIDER, Wolfgang; ARTELT, Cordula. Metacognition and mathematics education. ZDM: International Journal on Mathematics Education, Berlín, v. 42, n. 2, p. 149-161, 2010. 
SCHRAW, Gregory; CRIPPEN, Kent; HARTLEY, Kendall. Promoting self-regulation in science education: Metacognition as part of a broader perspective on learning. Research in Science Education, Amsterdam, v. 36, n. 1-2, p. 111-139, 2006.

SCHRAW, Gregory; DENNISON, Rayne. Assessing metacognitive awareness. Contemporary Educational Psychology, New York, v. 19, n. 4, p. 460-475, 1994.

SCHUNK, Dale. Metacognition, self-regulation, and self-regulated learning: research recommendations. Educational Psychology Review, New York, v. 20, n. 4, p. 463-467, dic. 2008.

SCHWONKE, Rolf et al. Metacognitive support promotes an effective use of instructional resources in intelligent tutoring. Learning and Instruction, Lovaina, v. 23, n. 1, p. 136-150, 2013.

SHEA, Nicholas et al. Supra-personal cognitive control and metacognition. Trends in Cognitive Sciences, Cambridge, v. 18, n. 4, p. 186-193, 2014.

SILVA, Onel. ¿Hacia dónde va la psicolingüística? Forma y Función, Bogotá, v. 18, p. 229-249, 2005.

SON, Lisa. Metacognitive control and the spacing effect. Journal of Experimental Psychology, Washington, DC, v. 36, n. 1, p. 255-262, 2010.

S00DLA, Piret; JÕGI, Anna Liisa; KIKAS, Eve. Relationships between teachers' metacognitive knowledge and students' metacognitive knowledge and reading achievement. European Journal of Psychology of Education, Amsterdam, v. 32, n. 2, p. 201-218, 2017.

SPRUCE, Robin; BOL, Linda. Teacher beliefs, knowledge, and practice of self-regulated learning. Metacognition and Learning, New York, v. 10, p. 245-277, 2015.

STERNBERG, Robert J. Foreword. In: HACKER, Douglas; DUNLOSKY, John; GRAESSER, Arthur (Ed.). Handbook of metacognition in education. New York: Routledge, 2009. p. 8-9.

TANNER, Kimberly. Promoting student metacognition. CBE Life Sciences Education, Bethesda, v. 11, n. 2, p. 113-120, 2012

THOMAS, Karen; BARKSDALE-LADD, Mary Alice. Metacognitive processes: teaching strategies in literacy education courses. Reading Psychology, London, v. 21, n. 1, p. 67-84, 2000.

TULVING, Endel; MADIGAN, Stephen. Memory and verbal learning. Annual Review of Psychology, Palo Alto, v. 21, n. 1, p. 437-484, 1970.

VAN DER STEL, Manita et al. The increasing role of metacognitive skills in math: a cross-sectional study from a developmental perspective. ZDM: International Journal on Mathematics Education, Berlín, v. 42, n. 2, p. 219-229, 2010.

VAN KRAAYENOORD, Christina. The role of metacognition in reading comprehension. In: TROLLDENIER, Hans-Peter; LENHARD, Wolfgang; MARX, Peter (Ed.). Focal points of the research and development of pedagogically-psychological perspectives. Göttingen: Hogrefe, 2010. p. 277-302. 
VAN LO0N, Mariëtte et al. Refutations in science texts lead to hypercorrection of misconceptions held with high confidence. Contemporary Educational Psychology, New York, v. 42, p. 39-48, 2015.

VEENMAN, Marcel. Giftedness: predicting the speed of expertise acquisition by intellectual ability and metacognitive skillfulness of novices. In: SHAUGHNESSY, Michael; VEENMAN, Marcel; KENNEDY KLEYN, Cynthia (Ed.). Meta-cognition: a recent review of research, theory, and perspectives. New York: New Science, 2008. p. 207-220.

VEENMAN, Marcel. Metacognition. In: AFFLERBACH, Peter (Ed.). Handbook of individual differences in reading, reader, text, and context. London: Routledge, 2016. p. 26-40.

VEENMAN, Marcel; VAN HOUT-WOLTERS, Bernadette; AFFLERBACH, Peter. Metacognition and learning: conceptual and methodological considerations. Metacognition and Learning, New York, v. 1, p. 3-14, 2006.

VINING, Alexander; MARSH, Heidi. Information seeking in capuchins (Cebus apella): a rudimentary form of metacognition? Animal Cognition, Berlín, v. 18, n. 3, p. 667-681, 2015.

WANG, Margaret; HAERTEL, Geneva; WALBERG, Herbert. Toward and knowledge base for school learning. Review of Educational Research, Washington, DC, v. 63, n. 3, p. 249-294, 1993.

WELLMAN, Henry. The origins of metacognition. In: FORREST PRESLEY, Donna; MACKINTON, George; WALLER, Gary (Ed.). Metacognition, cognition and human perfomance. Orlando: Academic Press, 1985. p. 1-30.

WHITEBREAD, David; PINO PASTERNAK, Deborah. Metacognition, self-regulation \& meta-knowing. In: LITTLETON, Karen; WOOD, Clare; KLEINE-STAARMAN, Judith (Ed.). International handbook of psychology in education. Bingley: Emerald, 2010. p. 673-712.

WINNE, Philip; AZEVEDO, Roger. Metacognition. In: SAWYER, R. Keith (Ed.). The Cambridge handbook of the learning sciences. Chapel Hill: Cambridge University Press, 2015. p. 63-87.

XU, Judy; METCALFE, Janet. Studying in the region of proximal learning reduces mind wandering. Memory \& Cognition, Madison, v. 44, n. 5, p. 681-695, 2016.

YEUNG, Nick; SUMMERFIELD, Christopher. Metacognition in human decision-making: confidence and error monitoring. Philosophical Transactions of the Royal Society of London. London, v. 367, n. 1594, p. 13101321 , 2012. Series B. Biological sciences.

ZALLA, Tiziana et al. Metacognition of agency and theory of mind in adults with high functioning autism. Consciousness and Cognition, San Diego, v. 31, p. 126-138, 2015.

ZEPEDA, Cristina et al. Direct Instruction of metacognition benefits adolescent science learning, transfer, and motivation: an in vivo study. Journal of Educational Psychology, Washington, DC, v. 107, n. 4, p. 954-970, 2015. 
Recibido en: 07.11.2017

Revisiones en: 24.04 .2018

Aprobado en: 13.06.2018

Ángel Valenzuela M. Doctor (c) en Ciencias Humanas, Universidad de Talca. Student Doctoral del Centro de Investigación en Ciencias Cognitivas, Universidad de Talca. Becario CONICYTPFCHA/Doctorado Nacional/2017-21171977. 